\title{
Familial Occurrence of Myxoedema
}

\author{
PETER F. HALL* \\ From the Department of Endocrinology, Guy's Hospital, London
}

During a period of 18 years, 38 patients suffering from myxoedema were treated at the Endocrine Clinic of Guy's Hospital, and the present paper is a report of the genetic aspects in these patients.

\section{Subjects and Methods}

This group of patients does not include examples of cretinism, nor of hypothyroidism of pituitary origin or that following thyroidectomy. Patients referred to the clinic as possible cases of hypothyroidism but who were found on careful observation not to meet the criteria referred to below were not included. Moreover, prolonged ingestion of iodides (c.g. in asthma) and treatment with resorcinol and other drugs that are known to cause hypothyroidism (Bull and Fraser, 1950; Werner, 1955; Means, DeGroot, and Stanbury, 1963) were eliminated as possible aetiological factors in the present series of patients. All the patients were referred from outside the clinic, three from other clinics within the hospital, and the remainder from practitioners throughout England. At the time of this study there was no reason to suppose that any of the patients were referred because of the present interest in hereditary factors in myxoedema. In one of the families reported here (Family No. 3), the propositus (IV.7) attended the clinic because she began to suffer symptoms like those of her mother who was herself attending for treatment of myxoedema. In all other cases relatives suffering from thyroid disease were receiving treatment outside the hospital.

The question of whether these 38 patients constitute a representative group of cases of myxoedema or whether referral to an Endocrine Clinic constituted selection of an order likely to influence the incidence of hereditary factors among the group requires some consideration. Reasons for believing that they do not constitute a highly select or unusual group include the following.

In the first place none of the patients was referred specifically for eugenic reasons, e.g. advice about the likelihood of inheriting myxoedema. Secondly, there were no unusual features about the disease in any of the patients-they were all typical examples of myxoedema clinically, according to laboratory investigations and

Received March 23, 1965.

* Present Address: Department of Physiology, University of Pittsburgh, School of Medicine, Pittsburgh, Pennsylvania. in the response shown to thyroid extract. The age of onset and the ratio of males to females were typical of those of other series of patients suffering from myxoedema (Werner, 1955; Means et al., 1963). Thirdly, the referring practitioners were different for each of the 38 patients, suggesting that the patients were not from any special geographical area and were not referred by practitioners with a special interest in thyroid disease.

No doubt referral to an Endocrine Clinic does represent selection-perhaps advanced cases of the disease are more likely to be referred for teaching purposes. It is considered, however, that there is no reason to believe that this selection has brought to the clinic an unusual number of patients with affected relatives. Moreover since little is known of the familial occurrence of myxoedema, interest in the findings reported here is not diminished by the possibility that the present group of patients may show an unusual number with affected relatives.

In order to establish uniform and reproducible pedigrees it was necessary to define myxoedema for present purposes. It is believed that with the aid of a careful history, a detailed physical examination, appropriate laboratory tests, and observation during the early stages of thyroid therapy, it is possible to decide whether an individual is suffering from myxoedema at the time of examination or not, unless he is receiving maintenance doses of thyroid extract. With the aid of the methods given below a decision was reached in every patient and in those relatives that were successfully traced. So-called partial forms of myxoedema in which the disease is confined to some special features, e.g. infertility or obesity or loss of hair, in whom it is believed that the full syndrome would occur unless treatment were given, are not included here.

Examination of Patients. Each patient was submitted to the usual history and physical examination. Every patient was asked whether there were any cases of thyroid or other endocrine disorders in the family. Laboratory investigations included basal metabolic rate (BMR), serum cholesterol, and in certain cases protein-bound iodine (PBI). When the diagnosis of myxoedema was made the patient was observed during the first 4 weeks of treatment and the response was carefully studied. Laboratory investigations were repeated at various time intervals after treatment had begun. All 38 patients showed all of the following. 
(I) Typical history and clinical picture before treatment (or following withdrawal of trcatment); (2) low BMR; (3) high serum cholesterol; and (4) typical response of these changes to thyroid extract.

In some patients retrospective study of the Clinic records did not enable us to recognize these changes with confidence. When this was so, thyroid therapy was withdrawn for 4 weeks during which the patient was seen 3 times a week and the above changes, including the response to thyroid after the 4 th week, were observed. It has been found by other workers that, 4 weeks after withdrawal of thyroid, BMR in myxoedema falls to levels below normal and the symptoms of the disease are clearly recognizable (Werner, I955; Means et al., 1963).

Inquiry into Health of Relatives. It was believed that the answer to a simple question regarding the occurrence of thyroid disease in the family was quite inadequate for the present purpose. Accordingly after a decision concerning the patients' health had been reached and after a satisfactory response to treatment was observed, a series of special appointments (varying in number according to the needs in each case) was made to question each of the 38 patients in detail, regarding the health of the following members of their families. (i) Sibs; (ii) children; (iii) parents; (iv) sibs of parents. It was found that if appointments were made at times convenient to the patients and to their relatives, all were extremely co-operative and in full sympathy with the eugenic aspects of the problem.

One patient died before this investigation was started and his family was investigated with the help of his sister. The procedure adopted was altered to suit each family. In some cases relatives consented to visit the hospital in order to give information about other members of the family. In others visits were made to the house and a number of relatives, gathered for the occasion, could be seen at one time. Some relatives were less accessible and a carefully planned auestionnaire was prepared. The questions were designed to discover how many of the relatives were regularly attending a physician or hospital for any chronic illness. When the answers were regarded as of possible interest the physician or hospital was written to.

Diagnosis of Thyroid Disease in Relatives. Since some of the affected relatives could not be examined, it was necessary to establish the diagnosis with the aid of medical records from hospitals and practitioners. The clinical data given below for the affected relatives indicate how this was done in each case. A number of the relatives agreed to visit the hospital for examination and testing, with the consent of their medical attendants. When this was not possible it was required that evidence from medical records, describing (i) the syndrome before treatment and (ii) response to thyroid extract, be obtained before the diagnosis of myxoedema could be established. When these descriptions clearly indicated the existence of myxoedema, the diagnosis was accepted in retrospect without laboratory data. The diagnosis of hyperthyroidism and goitre was based upon clinical and laboratory evidence collected in the same way. Diagnosis? without laboratory data was made in 3 relatives out of I8-Family 2 (II.I), Family 4 (II.2), and Family $5 \stackrel{\text { ज) }}{\frac{9}{5}}$ (II.3).

Age of Onset of Myxoedema. Propositi and affected relatives were carefully questioned to establish $\underset{\Phi}{\Phi}$ as closely as possible the age at which myxoedema first appeared. When a detailed history taken at the ${ }^{\infty}$ time of onset of the disease was available this was used $\vec{\circ}$ to determine when the disease began. The only other method available was that of careful questioning. It $\vec{\omega}$ was useful to learn which symptom of the disease the patient found most distressing and to attempt to dis- $\overline{3}$ cover when this first appeared. It is realized that the gradual onset of symptoms, so common in myxoedema, makes any attempt to localize the first appearance of $\rightarrow$ the disease approximate at best.

Laboratory Methods. Basal metabolic rate was measured by the method of Robertson (Robertson and $\vec{c}$ Reid, 1952). The normal range was + 10 to $-10 \% . \Phi$

Serum cholesterol was estimated by a modification of the Liebermann-Burchard reaction (Foote and Meri- $\frac{\mathbb{D}}{3}$ vale, 1949). The normal range was found to be 129-342 $\mathrm{mg}$./100 ml. (mean of $248.3 \mathrm{mg}$. $/ 100 \mathrm{ml}$. and standard deviation $\pm 47 \cdot 8$ ). Serum protein-bound iodide was $\vec{\varphi}$ measured by the method of Barker and the normal range was 4.0 to $8.0 \mu \mathrm{g}$. per $100 \mathrm{ml}$. (de Mowbray apd. Tickner, 1952).

\section{Results}

Examination of Myxoedema Patients. Allő 38 patients fulfilled the criteria given above for the $\stackrel{\varrho}{\rightarrow}$ diagnosis of myxoedema. The aetiological factors $\overrightarrow{\overrightarrow{0}}$ already mentioned were carefully excluded and in 3 no case was any evidence of goitre found-thyroid tissue was impalpable in each case. In the 2 patients: with juvenile myxoedema in the series, care was taken to exclude the possibility of cretinism. In every patient the disease was quite typical of the 3 textbook descriptions. Of the 38 patients, 7 were male (sex ratio $I: 5$ ).

Relatives of Myxoedema Patients. Of the $\stackrel{\circ}{\supset}$ 248 relatives,* which made up the 38 families examined, 73 were seen by the author, 5I were? seen by another relative and the questionnaire $N$ answered satisfactorily, 6 answered the questions too vaguely to be of any value, and 22 were not contacted. The remaining relatives (96) were dead, $\omega$ and copies of death certificates were obtained in 75 of these. None of the death certificates ofo

* This figure includes only those relatives whose full name and: approximate whereabouts were known. 
those relatives about whom information was obtained in this way alone mentioned myxoedema, so that the 96 deceased relatives included no cases of proven myxoedema.

Among the relatives seen by the author it was clear that at the time of examination some either had myxoedema or were free of it. Partial forms or intermediate types between normal and affected were not encountered.

In none of the 38 families was consanguinity discovered-defined here as marriage between second cousins or between those more closely related.

Families with Affected Relatives. The following pedigrees are those of the 38 families in which it could be established that members other than the propositus were affected by thyroid disease. For this purpose thyroid disease included myxoedema, all forms of hyperthyroidism, and non-toxic goitre. The non-affected subjects appearing in these pedigrees are so designated either because they were seen by the author, or because information obtained by questionnaire was considered decisive. The number of such unaffected relatives shown in each pedigree was determined by considerations of space. The age given for each patient is that at the beginning of 1960. The members of each family are identified by the usual pedigree co-ordinates.
Family I (Fig. I).

\section{Case Reports}

Propositus (IV.2).-The patient, aged 28 years, was referred to Guy's Hospital at the age of 22 years for assessment of the dosage of thyroid then being prescribed. She was examined at the hospital 3 times a week for 4 weeks following the withdrawal of thyroid extract, and during this time she showed typical features of myxoedema confirming an earlier diagnosis made elsewhere. Four weeks after withdrawal of thyroid BMR was $-32 \%$, serum cholesterol $410 \mathrm{mg}$./100 ml., and PBI $2 \mu \mathrm{g}$. $/ 100 \mathrm{ml}$.

MOTHER's Cousin (III.I). She was seen, at 48 years, at Guy's Hospital and had been taking thyroid tablets for 9 years. She agreed to stop treatment for 4 weeks in order to confirm the diagnosis. During that time the typical clinical picture of myxoedema emerged. BMR at that time was $-28 \%$ and serum cholesterol $430 \mathrm{mg}$. $/ 100 \mathrm{ml}$.

DAUGHTER OF MOTHER's Cousin (IV.r). The patient, aged 18 , was seen privately by a consultant physician at the age of 16 years. The diagnosis of myxoedema was made on clinical grounds and confirmed by a BMR of $-36 \%$, and by a typical response to thyroid extract.

Family 2 (Fig. I).

Propositus (III.8). The patient, aged 58 years, was referred to Guy's Hospital for an opinion regarding her treatment. She had been taking thyroid tablets for 6 years and was examined during the first 4 weeks following withdrawal of this therapy. The typical features of myxoedema appeared during this time and these

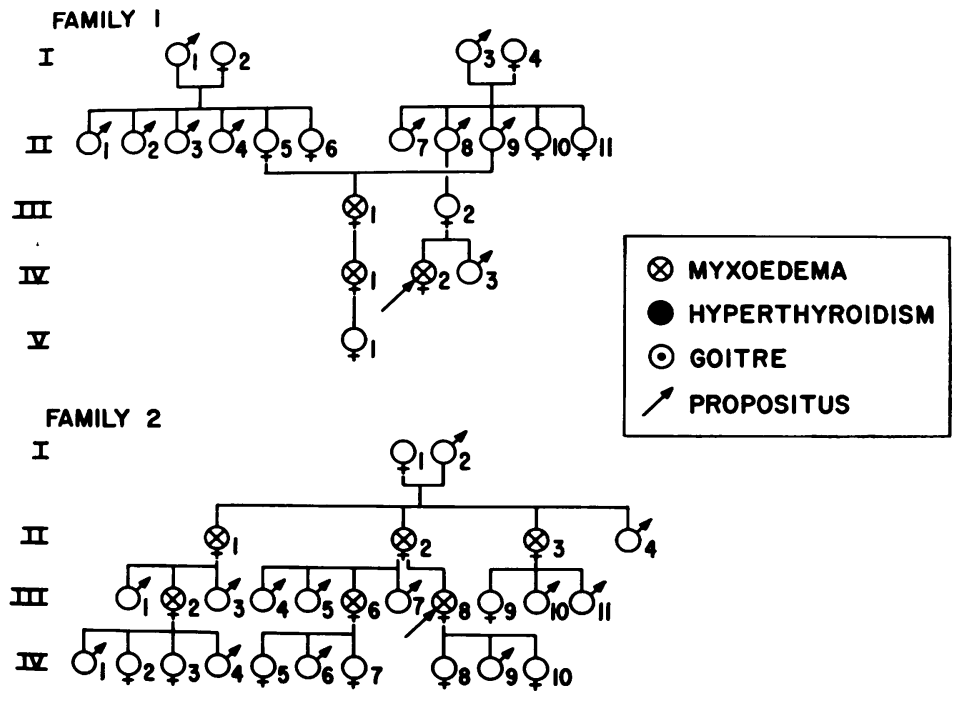

FIg. I. Two pedigrees showing the familial occurrence of myxoedema. 


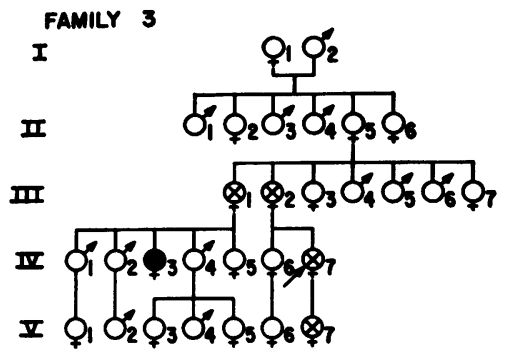

FAMILY 5

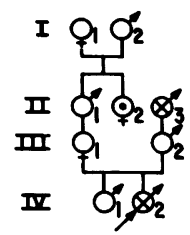

FAMILY 6
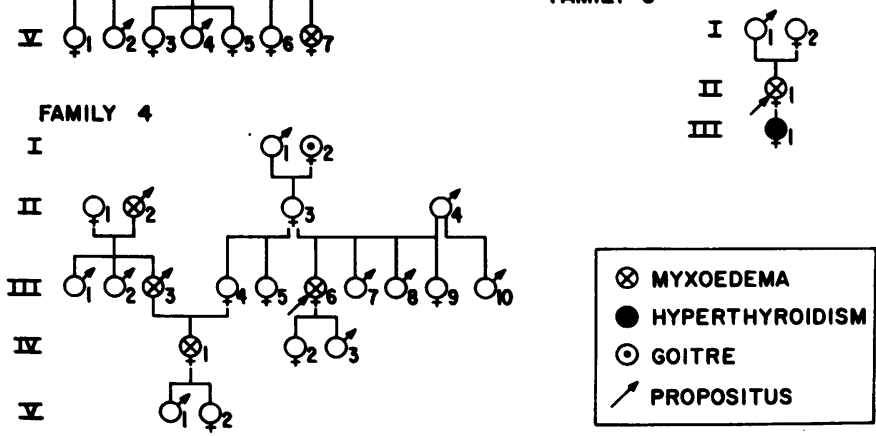

FIG. 2. Pedigrees showing the familial occurrence of thyroid disease.

symptoms promptly responded to thyroid therapy. Four weeks after stopping treatment, BMR was $-34 \%$, serum cholesterol was $398 \mathrm{mg}$./100 ml., and PBI was I $\mu \mathrm{g} . / \mathrm{I} 00 \mathrm{ml}$.

MOTHER (II.2). The patient's mother, aged 79 years, had taken thyroid for $2 \mathrm{I}$ years. A diagnosis of myxoedema was made by a consultant physician whose records showed typical symptoms of myxoedema with a classical response to thyroid. At the age of 64 years she stopped taking the tablets and was seen by a practitioner who described her as showing the typical features of myxoedema. BMR was $-22 \%$. She responded to thyroid extract when this was prescribed.

MOTHER's Elder Sister (II.I). This patient, now deceased, was seen at a teaching hospital, the records of which indicated that she showed typical signs of myxoedema and a classical response to thyroid therapy. She was seen by a general practitioner after she stopped taking the tablets for several weeks. He described her as 'typically myxoedematous' and stated that she responded to thyroid extract when this was prescribed.

MOTHER's YOUNGER SISTER (II.3). The patient, aged 46 years, was seen at the same teaching hospital as her eldest sister. The hospital records reveal that the typical clinical picture of myxoedema was observed: BMR was $-34 \%$. The patient responded satisfactorily to thyroid extract. She was later seen at Guy's Hospital and following withdrawal of thyroid for 4 weeks, the diagnosis was confirmed: $B M R$ was $-28 \%$ and serum cholesterol was $420 \mathrm{mg}$./ $100 \mathrm{ml}$. at the end of the fourth week.
DAUGHTER OF MOTHER's Elder Sister (III.2). The patient, aged 6I years, was examined at Guy's Hospitafo She had been taking thyroid extract for $20^{\circ}$ years was seen during a period of 4 weeks without treatment. Physical examination at that time revealed typical sign of myxoedema. BMR $-38 \%$ and serum cholesterof was $360 \mathrm{mg} . / 100 \mathrm{ml}$. The symptoms disappeare $\bar{\Phi}$ when treatment was resumed.

HALF-SISTER (III.6). The patient, now aged 39 years was examined at the age of 32 years by a consultant physician who diagnosed myxoedema and prescribed thyroid extract. The diagnosis was confirmed by with drawing thyroid for 4 weeks. During this time signs of myxoedema developed: $\mathrm{BMR}$ was $-34 \%$ and serum cholesterol $362 \mathrm{mg}$. $/ 100 \mathrm{ml}$. The patient returned normal when treatment was resumed.

Family 3 (Fig. 2).

Propositus (IV.7). The patient, aged 50, complained of symptoms suggesting myxoedema during the early months of her only pregnancy (aged 28 years). She was seen at Guy's Hospital and the diagnosis of myxoedent was made on clinical grounds and supported by a $B M R$. of $-39 \%$ though the serum cholesterol was $240 \mathrm{mg}$ 每 I00 $\mathrm{ml}$. The patient responded to thyroid extract and Io years later when this treatment was withheld for 4 weeks the diagnosis was confirmed by the appearance्d of symptoms of myxoedema: BMR $-42 \%$, cholesterd $4 \mathrm{I} 4 \mathrm{mg}$./ $100 \mathrm{ml}$, and by a return to normal when thyroid therapy was instituted.

MOTHER (III.2). The mother, aged 72 years, was seen at Guy's Hospital at the age of 53 when she complained 
of symptoms of myxoedema; the picture was considered typical of this disease and the diagnosis confirmed by response to thyroid extract. At the age of 60 she stopped taking thyroid and three weeks later was examined at Guy's Hospital where her BMR was found to be $-42 \%$ and serum cholesterol $380 \mathrm{mg}$./100 ml. She improved immediately after resuming thyroid extract.

AUNT (III.I). The patient, now deceased, showed typical features of myxoedema at the age of 48 , when she was seen at Guy's Hospital. The diagnosis was made on clinical grounds and confirmed by response to thyroid extract. When she was 64 she developed diabetes and her thyroid function was re-examined by withdrawing thyroid extract for 2 weeks. At this time her BMR was $-34 \%$ and serum cholesterol $408 \mathrm{mg}$./ I00 $\mathrm{ml}$. She again responded to thyroid extract.

DAughter (V.7). The patient, aged 22 years, complained of dry skin, constipation, puffiness of the face, and mental lethargy at the age of 15 years. She was then seen at Guy's Hospital where she was found to show the typical features of juvenile myxoedema. BMR was $-33 \%$ and serum cholesterol $440 \mathrm{mg} . / 100 \mathrm{ml}$. She responded dramatically to thyroid extract.

Mother's Cousin (IV.3). The patient, aged 42 years, developed acute thyrotoxicosis at the age of 26 years. At this time the diagnosis was made at a teaching hospital where $B M R$ was found to be $+32 \%$. The patient improved considerably with the aid of conservative therapy and when seen at Guy's Hospital at the age of 34 years she showed exophthalmos but appeared otherwise normal.

\section{Family 4 (Fig. 2).}

Propositus (III.6). The patient, aged 47 years, suffered symptoms of hypothyroidism from the age of 39 years when thyroid extract was prescribed. She was seen at Guy's Hospital at the age of $4 \mathrm{I}$ years and the diagnosis of myxoedema was confirmed by withdrawing treatment for 4 weeks. At the end of this time symptoms and signs of myxoedema were evident while the BMR was $-26 \%$ and serum cholesterol was $416 \mathrm{mg}$./ $/ 100 \mathrm{ml}$. The patient returned to normal when thyroid extract was recommended.

Maternal Grandmother (I.2). This patient, now deceased, was seen by a physician at the age of 50 and gave a history of swelling of the neck for 20-30 years. She was described as showing an obvious nodular goitre which had been treated by 'medicine' on and off for years but apparently thyroid function was normal. Her family physician confirmed this impression after referring to records kept by his predecessor.

HALF-SISTER'S HUSBAND (III.3). The patient, now aged 58 years, had at the age of 46 complained of fatigue, loss of memory, dry skin, and husky voice. He was examined at a teaching hospital where a diagnosis of myxoedema was made and confirmed by BMR of $-24 \%$ and serum cholesterol of $404 \mathrm{mg}$./ $100 \mathrm{ml}$.

FATHER OF HALF-SISTER's HusbaNd (II.2). This patient, now deceased, had at the age of 40 begun to complain of lethargy, dry skin, forgetfulness, constipation, and unaccustomed intolerance to cold. A series of family snapshots taken at this time strongly suggests myxoedema. The family physician prescribed thyroid extract and the patient's eldest son (like his father a pharmacist) described the response as dramatic. The records of the family physician (now in the hands of another practitioner) support this claim, as do further family portraits. The son recalled that some years later his father tried to do without thyroid extract but his symptoms returned. Myxoedema is mentioned in the death certificate.

HALF-Sister's DaUghter (IV.I). The patient, now 26 years, was taken at the age of 19 to a teaching hospital because of symptoms that suggested to her father that she too was suffering from myxoedema. The diagnosis was confirmed on clinical grounds and by a BMR $-28 \%$ and serum cholesterol $424 \mathrm{mg}$./ $100 \mathrm{ml}$. The patient responded favourably to thyroid extract.

\section{Family 5 (Fig. 2).}

Propositus (IV.2). The patient, now I8 years of age, was normal at birth and reached the usual milestones at the expected ages. He attended school from the age of 5 years and his performance there attracted no special attention. At the age of 8 he was found to have difficulty in learning, and he suffered from severe constipation, a dry scaly skin, and cold extremities. He was referred to Guy's Hospital where a clinical diagnosis of hypothyroidism was made. Serum cholesterol was $294 \mathrm{mg}$. $100 \mathrm{ml}$. The patient responded dramatically to thyroid extract-a response confirmed by careful photography of the patient's face and skin. At the age of 17 thyroid extract was withdrawn for three weeks and the symptoms of myxoedema returned. BMR was $-24 \%$ and PBI I $\mu \mathrm{g} . / 100 \mathrm{ml}$.

Paternal Grandfather (II.3). The patient, now aged 64, had consulted his family physician when he was 32 because of intolerance to cold, somnolence, loss of energy, constipation, dry skin, and loss of hair. The physician referred the patient to a city hospital where the diagnosis of myxoedema was made on clinical grounds and a gratifying response to thyroid therapy was observed. The mother of the propositus nursed the patient and described several relapses which occurred when the patient stopped taking thyroid extract.

Mother's Paternal Aunt (II.2). The patient, now 72 years, had suffered from a large goitre since puberty, which was clearly evident in several photographs and was confirmed by the family doctor who stated that thyroid function was normal on clinical examination. 
Family 6 (Fig. 2).

Propositus (II.I). This 53-year-old patient was referred to Guy's Hospital at the age of 49 years. History and physical examination revealed typical myxoedema: BMR - 33\%, serum cholesterol $424 \mathrm{mg}$./ $100 \mathrm{ml}$., and PBI 2.I $\mu \mathrm{g}$./ $/ 00 \mathrm{ml}$. She showed rapid improvement following the administration of thyroid extract.

DAUGHTER (III.I). The patient, now 28, suffered from hyperthyroidism of acute onset at the age of 2 I years. She was investigated at a city hospital where it was found that $\mathrm{BMR}$ was $+\mathbf{2 8 \%}$ and physical examination revealed typical hyperthyroidism. She responded favourably to subtotal thyroidectomy.

It will be seen that Families $1-5$ show more than one example of myxoedema each. The incidence of thyroid disease shown in Fig. I and 2 should be regarded as setting a minimum, since at least 4 additional relatives were alleged to have been treated for 'thyroid disease', but it was not possible to obtain sufficient evidence to include these people as established examples of thyroid disease.

Among these 5 families one propositus was male and among all those affected 4 of 16 were male, showing that the sex ratio was approximately the same among the whole series of 38 , among propositi of Families $\mathrm{I}-5$ and among affected relatives.

Further examination of Families I-5 reveals the following. (I) In 8 cases, myxoedema is seen in parent and child (Families I, 2, 3, and 4). (2) In 2 cases, sibs were affected (Families 2 and 3 ). (3) In one, an affected woman married twice, in each case a normal man, and had an affected child by each husband (Family 2). (4) In one case, myxoedema was seen on both sides of the family (Family 4). (5) In two families (Families 3 and 4) the disease appeared in 3 successive generations. (6) In one family (Family 5) myxoedema occurred in two generations skipping the intervening generation. In no case were husband and wife both affected.

Family 6 show myxoedema and thyrotoxicosis in mother and daughter. One example of thyrotoxicosis is seen in Family 3, and in Family 4 and Family 5 there was one example each of goitre with normal thyroid function.

\section{Discussion}

The 38 patients and their affected relatives who form the basis of this report are believed to represent typical examples of myxoedema. These patients do not include examples of hypothyroidism of known aetiology (e.g. that due to drugs, surgery, etc.). Moreover, the disease as manifested by thes? patients is not only typical of classical myxoedem but, so far as can be determined by careful clinicat examination and laboratory investigations, is uni $\overrightarrow{\overline{\bar{C}}}$ form in the manner of its expression, both within one family and from family to family. The ratiof of affected males to females and the age of onse每: of the disease are in keeping with findings by othe workers (Means et al., 1963; Werner, 1955).

It would greatly facilitate the evaluation of the present data, if the incidence of myxoedema iB the general population were known. The only information concerning this aspect of the diseases appears to be that reported by Bartels (I94I) This author selected a group of normal people i⿺辶乛龰 Denmark and arranged to obtain information abouin the health of their relatives by means of a question $\omega$ naire. He found among 3,200 relatives 3 cases of probable myxoedema of which one appeared cero tain. If we accept all 3 as examples of myxoedema? the incidence of the disease would be approximately I per I000 head of population. The study of Bartels was primarily concerned with hyper thyroidism and he found this disease to be $4 \cdot$ 로 $^{\circ}$ times more common than myxoedema. In th® absence of similar studies in Great Britain the findings of Bartels will be regarded as applyingsto the population from which the present patients were taken. It may be pointed out that at the Endoctîn Clinic of Guy's Hospital the incidence of hypers thyroidism was 5 times greater than that of myxoe edema, in keeping with the observations of Bartel: in the Danish population.

The families reported here include 210 member excluding the propositi. The probability that $21 \odot$ people would include two examples of a disease that affects I per rooo head of the population is. $(0.210)^{2}$ or 0.044 . It was noted that the 2 I relatives included I I cases of myxoedema. It $\mathrm{i}$, clear that the possibility of such a finding being the result of chance can be dismissed.

Under these circumstances the possibility tha myxoedema is an hereditary disease must be considered. Examination of the present pedigree? (Fig. I and 2) reveals that certain forms of inheri $>$ tance can be dismissed at once. For examp simple dominance, sex-linked dominance, and sex-influenced dominance each fails to accoun? satisfactorily for the occurrence of myxoedema in these families. Moreover it is unlikely that sex linked recessivity could alone account for these findings. However, simple recessivity and sexo influenced recessivity cannot be excluded. Thह following observations are compatible with eithe? of these possibilities. 
Family I: The second generation was entirely normal and two brothers of this generation had affected granddaughters. One brother had an affected daughter.

Family 2: In this family 3 sisters were affected.

Family 3: Two sisters were affected.

Family 4: Both sides of the family were affected.

Family 5: In this family a normal man was both the son of an affected father and the father of an affected son.

However, there are at least two observations that are difficult to reconcile with either simple recessivity or sex-influenced recessivity.

Family 2: Half-sisters were affected (III.6 and III.8).

Family 4: Myxoedema appeared in three successive generations (II, III, and IV). However in the third generation (III) marriage with a man from an affected family occurred.

In a disease occurring in the general population as infrequently as myxoedema, the probability of a woman marrying two heterozygous normal men is extremely remote. Nevertheless, the findings do not entirely exclude recessive inheritance. Moreover, since myxoedema is at least 4 times as common in women as in men (Bartels, 194I; Werner, 1955) and in view of the fact that reproductive activity in women is known to involve episodes of thyroid stimulation (Means et al., 1963) it is probable that if hereditary factors do operate in this disease these factors will be sexinfluenced. The present data do not, therefore, exclude the possibility of sex-influenced recessivity.

Multifactorial inheritance, due either to the fact that several genes are required for the phenotypic expression of myxoedema or to the fact that the disease is an extreme variant of normal resulting from a specially unfavourable combination of genes, seems unlikely in view of the fact that partial forms of the disease or individuals intermediate between normal and affected were not found.

In view of these reservations regarding recessive inheritance of myxoedema, it is necessary to examine the possible role of environmental factors in the aetiology of the disease. There are two considerations that suggest that environmental factors may be important in myxoedema. In the first place a number of drugs have been reported to cause myxoedema (Means et al., 1963), and it is possible that others have yet to be discovered. It is not difficult to see how a drug could cause myxoedema in several members of one family. For example, resorcinol is used in the treatment of varicose ulcers and has been reported to cause myxoedema
(Bull and Fraser, 1950). It is conceivable that several women in one family, perhaps attending the same physician, could receive resorcinol for varicose ulcers. Although resorcinol has been eliminated as an aetiological factor in the present patients, it is possible that some other unknown environmental factor could be involved.

Some support for this possibility is found in a paper by Goldberg (1960) who reported a high incidence of myxoedema in certain families. The commonest finding was that both husband and wife were affected. Goldberg suggested the possibility of an insidious virus infection. In no case were husband and wife affected in the present series. Nevertheless it is clear that environmental factors must be carefully considered in the aetiology of myxoedema.

The question arises as to whether the observations reported here point to the existence of a special type of myxoedema in which heredity is an aetiological factor. Although myxoedema is aetiologically heterogeneous, careful inquiry and examination failed to reveal any special feature of the disease that was peculiar to the present families.

It is, therefore, concluded that the present families show a high incidence of myxoedema and that the occurrence of the disease in these families may reflect the operation of hereditary factors. The two most probable modes of inheritance, simple recessivity and sex-influenced recessivity, cannot be excluded by the data presented. Moreover, there is reason to believe that certain environmental factors, as yet undefined, may be important in the aetiology of the disease. Of the two possible forms of inheritance sex-influenced recessivity is the more likely.

The number of relatives showing hyperthyroidism or goitre in these families is too small to allow statistical evaluation. However the occurrence of these two conditions in the families discussed is in keeping with the suggestion of Bartels (I94I) that goitre, myxoedema, and hyperthyroidism may be related genetically. It is interesting to note that if this is so Family 5 can be regarded as showing bilateral inheritance of thyroid disease and thus supporting the possibility of recessive inheritance.

\section{Summary}

In a series of 38 patients suffering from proven myxoedema, a careful search revealed that 5 had relatives with myxoedema. The 5 families included I4 patients suffering from myxoedema in addition to the 5 propositi. A sixth propositus had a daughter with thyrotoxicosis. Another of the 5 families included a patient with thyrotoxicosis and in 
each of two families one relative suffered from non-toxic goitre. It is concluded that the present findings are compatible with sex-influenced recessive inheritance of myxoedema in these families and with a previous suggestion that hyperthyroidism and non-toxic goitre may be genetically related to myxoedema. In addition however, environmental factors may play an important role in the aetiology of myxoedema.

The author wishes to thank Dr. P. M. F. Bishop of Guy's Hospital for advice and encouragement during the performance of these studies and Dr. J. Howard Turner of the Department of Biostatistics, Graduate School of Public Health, University of Pittsburgh, Pittsburgh, Pennsylvania, for invaluable assistance during the preparation of this manuscript.

\section{REFERENCES}

Bartels, E. D. (I94I). Heredity in Graves' Disease, with Remarks on Heredity in Toxic Adenoma in the Thyroid, Non-toxic Goitre, and Myxoedema. E. Munksgaard, Copenhagen. (Thesis, Copenhagen, first published in Nord. Med., 3, 2908, 1939.)

Bull, G. M., and Fraser, R. (I950). Myxoedema from resorcinol ointment applied to leg ulcers. Lancet, $1,85 \mathrm{I}$.

de Mowbray, R. R., and Tickner, A. (1952). The diagnostic value of estimations of protein-bound iodine in serum. ibid., 2, 5 II.

Foote, J. B., and Merivale, W. H. H. (1949). Value of clinical laboratory tests. I. The concentration of cholesterol in the serum of patients with disorders of the thyroid gland. Guy's Hosp. Rep., 98, 202.

Goldberg, M. (I960). Incidence of hypothyroidism in more than one member of a family. Amer. F. med. Sci., 239, 562.

Means, J. H., DeGroot, L. J., and Stanbury, J. B. (1963). The Thyroid and its Diseases. McGraw-Hill Book Co., New York.

Robertson, J. D., and Reid, D. D. (I952). Standards for the basal metabolism of normal people in Britain. Lancet, 1,940 .

Werner, S. C. (1955). The Thyroid. Paul B. Hoeber, New York (2nd ed., 1962.) 